Address correspondence to: Lynn B. Dustin, Center for the Study of Hepatitis C, Laboratory of Virology and Infectious Disease, The Rockefeller University, 1230 York Ave., Box 64, New York, New York 10065, USA. Phone: 212.327.7067; Fax: 212.327.7048; E-mail: dustinl@rockefeller.edu.

1. Lavanchy D. The global burden of hepatitis C. Liver Int. 2009;29(suppl 1):74-81.

2. Afdhal NH. The natural history of hepatitis C. Semin Liver Dis. 2004;24(suppl 2):3-8.

3. Heathcote EJ. Antiviral therapy: chronic hepatitis C. J Viral Hepat. 2007; 14(suppl 1):82-88.

4. Casrouge A, et al. Evidence for an antagonist form of the chemokine CXCL10 in patients chronically infected with HCV. J Clin Invest. 2011; 121(1):308-317.

5. Butera D, et al. Plasma chemokine levels correlate with the outcome of antiviral therapy in patients with hepatitis C. Blood. 2005;106(4):1175-1182.

6 . Romero AI, et al. Interferon (IFN)- $\gamma$-inducible protein 10: association with histological results, viral kinetics, and outcome during treatment with pegylated interferon- $\alpha-2 \mathrm{a}$ and ribavirin for chronic hepatitis C virus infection. J Infect Dis. 2006 194(7):895-903.

7. Lagging $M$, et al. IP-10 predicts viral response and therapeutic outcome in difficult-to-treat patients with HCV genotype 1 infection. Hepatology. 2006;
44(6):1617-1625.

8. Diago M, et al. Association of pretreatment serum interferon- $\gamma$ inducible protein levels with sustained virological response to peginterferon plus ribavirin therapy in genotype 1 -infected patients with chronic hepatitis C. Gut. 2006;55(3):374-379.

9. Qin S, et al. The chemokine receptors CXCR3 and CCR5 mark subsets of $\mathrm{T}$ cells associated with certain inflammatory reactions. J Clin Invest. 1998;101(4):746-754.

10. Oo YH, Adams DH. The role of chemokines in the recruitment of lymphocytes to the liver.J Autoimmun. 2010;34(1):45-54.

11. Narumi S, et al. Expression of IFN-inducible protein-10 in chronic hepatitis. J Immunol. 1997; 158(11):5536-5544.

12. Harvey CE, et al. Expression of the chemokine IP-10 (CXCL10) by hepatocytes in chronic hepatitis C virus infection correlates with histological severity and lobular inflammation. J Leukoc Biol. 2003;74(3):360-369.

13. Shields PL, Morland CM, Salmon M, Qin S, Hubscher SG, Adams DH. Chemokine and chemokine receptor interactions provide a mechanism for selective $T$ cell recruitment to specific liver compartments within hepatitis C-infected liver. J Immunol. 1999;163(11):6236-6243.

14. Tan AT, et al. A longitudinal analysis of innate and adaptive immune profile during hepatic flares in chronic hepatitis B. J Hepatol. 2010;52(3):330-339.

15. Nishioji K, et al. Increase of chemokine interferon-inducible protein-10 (IP-10) in the serum of patients with autoimmune liver diseases and increase of its mRNA expression in hepatocytes. Clin Exp Immunol. 2001;123(2):271-279.

16. Rehermann B. Hepatitis $C$ virus versus innate and adaptive immune responses: a tale of coevolution and coexistence. J Clin Invest. 2009;119(7):1745-1754

17. Proost P, et al. Amino-terminal truncation of CXCR3 agonists impairs receptor signaling and lymphocyte chemotaxis, while preserving antiangiogenic properties. Blood. 2001;98(13):3554-3561.

18. Andrieu T, et al. Similar increased serum dipeptidyl peptidase IV activity in chronic hepatitis $C$ and other viral infections. J Clin Virol. 2003;27(1):59-68.

19. Zeremski M, et al. Intrahepatic levels of CXCR3-associated chemokines correlate with liver inflammation and fibrosis in chronic hepatitis C. Hepatology. 2008;48(5):1440-1450.

20. Zekry A, McHutchison JG, Diehl AM. Insulin resistance and steatosis in hepatitis $\mathrm{C}$ virus infection. Gut. 2005;54(7):903-906.

21. Mehta SH, Brancati FL, Sulkowski MS, Strathdee SA, Szklo M, Thomas DL. Prevalence of type 2 diabetes mellitus among persons with hepatitis C virus infection in the United States. Ann Intern Med. 2000;133(8):592-599.

22. Itou $\mathrm{M}$, et al. Altered expression of glucagon-like peptide- 1 and dipeptidyl peptidase IV in patients with HCV-related glucose intolerance.J Gastroenterol Hepatol. 2008;23(2):244-251.

23. Antonelli A, Ferri C, Ferrari SM, Colaci M, Sansonno D, Fallahi P. Endocrine manifestations of hepatitis C virus infection. Nat Clin Pract Endocrinol Metab. 2009;5(1):26-34.

\title{
Cytokinesis failure and attenuation: new findings in Fanconi anemia
}

\author{
Philip J. Mason and Monica Bessler
}

Division of Hematology, The Children's Hospital of Philadelphia, and University of Pennsylvania, Philadelphia, Pennsylvania, USA.

\begin{abstract}
The hallmarks of the rare inherited disorder Fanconi anemia (FA) are progressive bone marrow failure and susceptibility to cancer. The former is the major cause of death for patients with FA, as it usually occurs earlier in life than cancer development. Despite spectacular advances in unraveling the molecular details of FA, the origin of the bone marrow failure that is central to this condition for most patients has long been puzzling and controversial. Two studies recently published in the JCI, including one in this issue, will add to the debate. They also highlight the fact that studying rare disorders can elucidate important new clinical and biological principles.
\end{abstract}

Fanconi anemia (FA) is a recessive, inherited disorder that occurs with an incidence of approximately 1 per 350,000 births, that is, relatively rarely. One of the major hallmarks of FA is bone marrow failure (BMF), which is the major caused of death in patients with FA, but it is also associated with an increased susceptibil-

Conflict of interest: The authors have declared that no conflict of interest exists.

Citation for this article: J Clin Invest. 2011; 121(1):27-30. doi:10.1172/JCI45619. ity to leukemia and certain cancers (1). FA is caused by mutations in one of fourteen or more genes (the so-called FA genes), whose products cooperate to ensure the repair of interstrand crosslinks that form during DNA replication (i.e., during the $\mathrm{S}$ phase of the cell cycle) (2). Failure to repair these lesions efficiently leads to the activation of $S$ and $G_{2}$ cell cycle checkpoints that trigger cell cycle arrest $(3,4)$. Failure of cells, particularly HSCs and blood cell precursors, to progress through the cell cycle has been thought to contribute to the BMF experienced by patients with FA (5). Despite intensive investigation of the nature and function of the FA genes, the precise mechanism(s) underlying BMF and cancer susceptibility in FA has not been fully elucidated and remains controversial. The same can be said of other inherited BMF syndromes, such as dyskeratosis congenita, Diamond-Blackfan anemia, and Shwachman-Diamond syndrome (6). In these diseases, BMF develops slowly over the course of many years, making the process difficult to investigate in a cell line or even in a short-lived mouse model.

Two remarkable studies recently published in the JCI, including one in this issue, report findings that will change the way we think about BMF in FA and may eventually change the way patients with the disease are cared for and treated $(7,8)$. In a recent issue of the JCI, Vinciguerra et al. (7) reported that some FA cells proceed past the $\mathrm{G}_{2}$ checkpoint with DNA that is 

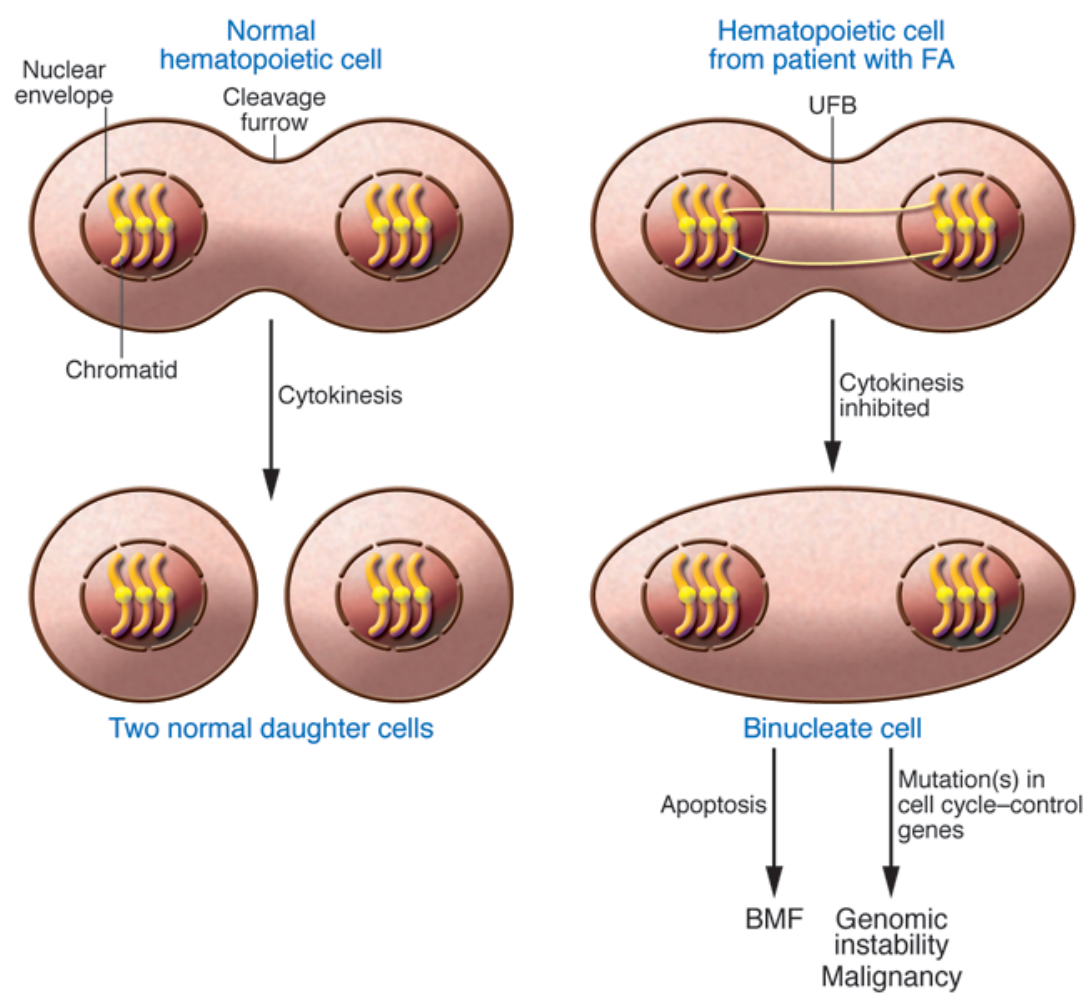

incompletely replicated and complete mitosis with sister chromatids in daughter nuclei still connected by ultrafine DNA bridges. It is at the cytokinesis stage, the point of division of the cytoplasm into two daughter cells, that problems arise. As reported in the second paper (8), which is published in this issue of the JCI, Ceccaldi et al. found that a proportion of FA patients, who live longer than others and do not have severe BMF, have developed a clone of blood cells that is still deficient in repairing interstrand crosslinks but no longer activates the $G_{2}$ checkpoint to trigger cell cycle arrest. This is due to a somatically acquired dampening of the signaling pathway that regulates the $\mathrm{G}_{2} / \mathrm{M}$ transition, although the precise molecular change was not defined.

\section{Failure of cytokinesis as a possible mechanism of BMF}

The impetus for the study by Vinciguerra et al. (7) was the recent finding that chromatin foci containing monoubiquitinated FA, complementation group D2 (FANCD2) and FANCI, which assemble during $\mathrm{S}$ phase and disassemble late in $S$ phase when DNA repair is complete, sometimes persist during and after mitosis at fragile sites in which DNA replication is slow $(9,10)$. In these cases, the sites in sister chromatids in which these foci are located are connected by ultrafine bridges (UFBs). These bridges are coated by two DNA helicases: Bloom syndrome, RecQ helicase-like (BLM), the protein mutated in Bloom syndrome (a rare autosomal recessive disorder characterized by a high frequency of chromosomal breaks and rearrangements), and Plk1-interacting checkpoint helicase (PICH), a checkpoint helicase (11). Crosslinking agents, such as mitomycin C (MMC), to which FA cells are exquisitely sensitive, increase the number of UFBs.

These findings led Vinciguerra et al. to study the effect of FA mutant phenotypes on the behavior of UFBs (7). They found that the number of UFBs was increased in fibroblasts and cell lines from patients with FA and in HeLa cells depleted of specific FA proteins. They went on to show that late in mitosis the UFBs were coated with the FA protein FANCM, which is important in recruiting the FA core complex to the sites of crosslinks and in facilitating the monoubiquitination of FANCD2 and FANCI. FANCM mutant cell lines do not ubiquitinate FANCD2 and are MMC sensitive (12). Interestingly, a FANCM mutant that does not have ATPase activity corrected the ubiquitination defect but not the MMC sensitivity

\section{Figure 1}

Cytokinesis failure in FA. PICH-BLM UFBs are ultrafine DNA bridges that have FANCD2$\mathrm{FANCl}$ foci at their extremities and are most likely derived from unresolved replication intermediates. The paper by Vinciguerra et al. (7) shows that hematopoietic cells from patients with FA have an increased number of UFBs between daughter nuclei that may inhibit cytokinesis, leading to binucleated or multinucleated cells. These cells may undergo apoptosis, contributing to BMF, or may lead to genomic instability and malignancy. or the increased number of UFBs, suggesting that ATPase activity is needed for preventing or resolving the bridges. The consequence of the increased number of UFBs is an increased number of binucleate or multinucleate cells arising from cytokinesis failure. Multinucleate cells were also generated by knock down of several FA proteins. BLM knock down and the FANCM ATPase mutation also result in an increase in the number of multinucleate cells (13). Can cytokinesis failure contribute to BMF in FA? Mice null for Fancy or Fancd2 show a HSC defect but not overt BMF. HSCs purified from these mice showed a higher percentage of multinucleate cells than did controls. Moreover, bridges joining the nuclei stained with lamin-associated polypeptide 2 , a nuclear membrane protein that accumulates around chromatin at the end of mitosis. The fate of cells failing cytokinesis is either apoptosis or cell cycle arrest and senescence. In the mice, an increase in apoptosis correlated with the increase in multinucleate cells (Figure 1).

This body of work reported by Vinciguerra and colleagues (7) highlights the importance of the FA proteins in cell division, in which they are shown to be important in the resolution of UFBs that arise from unresolved replication intermediates. 


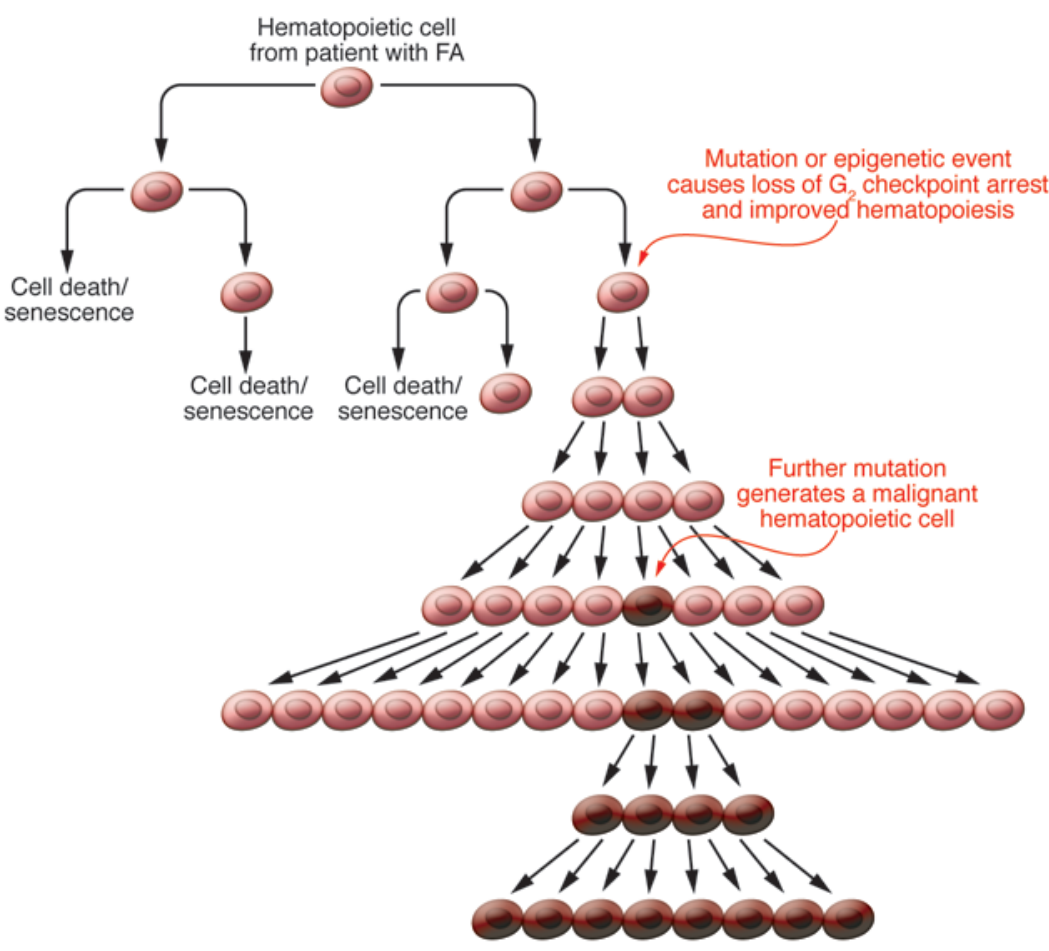

The question now is how important is the observed failure of cytokinesis in BMF or in progression to cancer? FANC mutant cells show increased chromosomal breaks at fragile sites (14), providing a connection between UFBs and genomic instability. If genomic instability or multinucleated cells result in apoptosis in HSCs, as Vinciguerra and colleagues observed (7), UFBs could contribute to BMF. Furthermore, if the cells are allowed to progress by somatic mutations compromising checkpoint regulation, this may lead to the development of cancer. However, BLM-deficient cells behave like the FA cells, in terms of the formation of UFBs and multinucleate cells, and patients with Bloom syndrome do not develop BMF. This implies that there may be a qualitative difference in the resolution of UFBs in Bloom syndrome versus FA, due to the presence or absence of FANC proteins at the UFB termini; alternatively, there might be an intrinsic difference in the downstream response, as discussed by Vinciguerra and colleagues (7).

\section{Attenuation of BMF for the price of leukemia later in life}

The study by Ceccaldi et al. began with the observation that a small number of patients have only two of the usual three criteria for FA (8). Like those of other FA patients, the peripheral blood cells (stimulated lymphocytes) of these patients lacked the crucial monoubiquitination of FANCD2 and showed increased chromosomal breaks after MMC treatment. But, unlike the cells from most FA patients, they did not show the characteristic $G_{2}$ arrest after MMC treatment. A cohort of 97 patients was examined in this way, and this phenotype was seen in 17 patients. Most of these patients were among the oldest in the cohort, their blood counts were almost normal, and five of them developed myelodysplastic syndrome (MDS) or acute myeloid leukemia (AML). The fact that this "attenuation" of the $\mathrm{G}_{2}$ checkpoint in response to DNA damage was an acquired characteristic was apparent, because the MMC-induced $\mathrm{G}_{2}$ arrest was still operational in fibroblasts from the same patients and because two patients were observed to convert to the attenuated phenotype from typical FA over the course of the analysis.

Attenuation was accompanied by clonal hematopoiesis, as demonstrated by a biased $\mathrm{X}$-inactivation (8), implying that all peripheral blood cells derived from a single progenitor cell in which a molecular event took place, giving rise to attenuated $\mathrm{G}_{2}$ arrest (Figure 2). However, three out of six classical FA patients also showed clonal hematopoiesis, as is com-

\section{Figure 2}

Clonal attenuation of a $G_{2}$ checkpoint in $F A$. Hematopoietic cells from patients with $F A$ exhibit slow growth and accumulate in $G_{2}$, leading to BMF. Ceccaldi et al. (8) show that, in some patients, blood counts are normalized due to the appearance of a clone of cells, in which the arrest at the $G_{2}$ checkpoint does not take place. Hematopoiesis in these patients is greatly improved, but the trade off is an increased susceptibility to later development of MDS and AML. This scenario, identified in FA, is likely to be a theme, applicable not only to many forms of inherited BMF syndromes but possibly to other regenerative disorders associated with an increased risk of malignant transformation. monly seen in individuals with inherited BMF syndromes (15). Arrest at the $G_{2}$ checkpoint is modulated by a pathway involving ataxia telangiectasia and Rad3 related (ATR) phosphorylation, p53 stabilization, and CHK1 checkpoint homolog (CHK1) expression (16). p53 and CHK1 levels were low in attenuated cells from six out of the nine patients with this phenotype, and CHK1 mRNA levels were low in attenuated cells from most of them. The search for the molecular explanation did not come up with any mutation or deletion in the P53 or CHK1 genes or any alterations in their methylation pattern. However, of two micro RNAs previously shown to target CHK1 expression, one of them, miR-15a, was present at a higher level in attenuated patients. That this might be important in the attenuation mechanism was supported by the authors' demonstration that CHK1 had a higher turnover, as assessed by proteosome inhibition studies, in attenuated cells (8). Verification that FA-induced arrest at the $G_{2}$ checkpoint can be regulated by the ATR and CHK1 pathway was provided by experiments with a HeLa cell line with a silenced FANCD2 gene. Knocking down ATR or CHK1 in these cells removed the arrest at the $\mathrm{G}_{2}$ checkpoint. A CHK1 inhibitor was also shown to attenuate EBV lines from patients with the classi- 
cal FA phenotype, while a CHK2 inhibitor had no effect. These cells were also protected from MMC-induced death by the CHK1 inhibitor. The cost of the attenuated FA phenotype in these patients was a higher risk of malignancy. Of the 97 patients in the cohort, five had excess blasts or overt myeloid leukemia. All of these had the attenuated phenotype. In four of these patients, blasts could be purified, and they all had very low CHK1 levels. Interestingly, low CHK1 levels are characteristic of AML generally, not only when arising from FA (17).

There are several important aspects of this work by Ceccaldi et al. (8). The attenuation phenotype may explain the very different clinical outcomes sometimes seen in two members of the same family who carry the same causative mutation, particularly in the absence of any evidence of genetic reversion (18). In addition, attenuation, identified by the dissociation of chromosomal breakage and $\mathrm{G}_{2}$ arrest after exposure to DNA crosslinking agents, is associated with a higher chance of transformation to malignancy, thus identifying patients who, although milder in their presentation of BMF, might need continuous, intensified tumor surveillance. Finally, the study by Ceccaldi et al. (8) identifies a druggable target, the $\mathrm{G}_{2}$ checkpoint, that, when suppressed, improves blood cell production and may prolong the survival of FA patients if the increased incidence of cancer can be controlled. If the checkpoint could be activated or restored, it could, in principle, be used to limit the growth of cancer cells by inducing cell cycle arrest and cell death.

\section{Conclusions and prospects}

Most previous studies have focused on the role of the FANC proteins in S phase. The papers by Vinciguerra et al. (7) and Ceccaldi et al. (8) provide important insights into different aspects of FA pathogenesis that converge on events in the $\mathrm{G}_{2}$ phase of the cell cycle and during mitosis. Vin- ciguerra et al. showed that in FA there is an increase in the number of cells that enter mitosis with incompletely replicated DNA and that this can lead to the formation of multinucleated cells. These, in turn, can lead to BMF by apoptosis or to genomic instability and cancer. Ceccaldi et al. showed that, in FA patients, clones of blood cells can arise in which the $G_{2}$ checkpoint has been attenuated and no longer slows cell cycle progression. This leads to an improvement in blood counts and lifespan but carries with it an increased risk of malignancy. Both papers illustrate that studying a rare disease can lead to the discovery of important clinical and biological principles, with implications far beyond the original investigation. In this case, DNA repair proteins are shown to be involved in the resolution of a new kind of ultrafine DNA bridge that, if unresolved, is associated with cytokinesis failure and cell death, providing a possible additional mechanism for BMF. The Ceccaldi paper, on the contrary, demonstrates that overcoming a cell cycle checkpoint is associated with an improvement in BMF, highlighting the importance of the activated $\mathrm{G}_{2}$ checkpoint in the pathogenesis of BMF.

\section{Acknowledgments}

Philip J. Mason's work is supported in part by NIH/NCI grant R01 CA106995, and Monica Bessler's work is supported in part by NIH/NCI grant R01 CA105312.

Address correspondence to: Philip J. Mason, Room 302E, Leonard and Madlyn Abramson, Pediatric Research Center, The Children's Hospital of Philadelphia, 3615 Civic Center Boulevard, Philadelphia, Pennsylvania 19104-4318, USA. Phone: 267.426.9327; Fax: 215.590.4834; E-mail: masonp@email.chop.edu.

\footnotetext{
1. Rosenberg PS, Greene MH, Alter BP. Cancer incidence in persons with Fanconi anemia. Blood. 2003;101(3):822-826.

2. de Winter JP, Joenje H. The genetic and molecu-
}

lar basis of Fanconi anemia. Mutat Res. 2009; 668(1-2):11-19.

3. Sabatier L, Dutrillaux B. Effect of caffeine in Fanconi anemia. I. Restoration of a normal duration of G2 phase. Hum Genet. 1988;79(3):242-244.

4. Akkari YM, Bateman RL, Reifsteck CA, D’Andrea $\mathrm{AD}$, Olson $\mathrm{SB}$, Grompe M. The 4N cell cycle delay in Fanconi anemia reflects growth arrest in late $\mathrm{S}$ phase. Mol Genet Metab. 2001;74(4):403-412.

5. Muller LU, Williams DA. Finding the needle in the hay stack: hematopoietic stem cells in Fanconi anemia. Mutat Res. 2009;668(1-2):141-149.

6. Bessler M, Mason P, Link D, Wilson D. Inherited bone marrow failure syndromes. In: Orkin S, Nathan D, Ginsburg D, Look A, Fisher D, Lux $\mathrm{S}$, eds. Nathan and Oski's Hematology of Infancy and Childhood. Philadelphia, Pennsylvania, USA: Saunders College Publishing; 2008:307-395.

7. Vinciguerra P, Godinho SA, Parmar K, Pellman D, D'Andrea AD. Cytokinesis failure occurs in Fanconi anemia pathway-deficient murine and human bone marrow hematopoietic cells. J Clin Invest. 2010;120(11):3834-3842.

8. Ceccaldi R, et al. Spontaneous abrogation of the $\mathrm{G}_{2}$ DNA damage checkpoint has clinical benefits but promotes leukemogenesis in Fanconi anemia patients. J Clin Invest. 2011;121(1):184-194.

9. Chan KL, Palmai-Pallag T, Ying S, Hickson ID. Replication stress induces sister-chromatid bridging at fragile site loci in mitosis. Nat Cell Biol. 2009;11(6):753-760.

10. Naim V, Rosselli F. The FANC pathway and BLM collaborate during mitosis to prevent micro-nucleation and chromosome abnormalities. Nat Cell Biol. 2009;11(6):761-768.

11. Chan KL, North PS, Hickson ID. BLM is required for faithful chromosome segregation and its localization defines a class of ultrafine anaphase bridges. EMBO J. 2007;26(14):3397-3409.

12. Singh TR, et al. Impaired FANCD2 monoubiquitination and hypersensitivity to camptothecin uniquely characterize Fanconi anemia complementation group M. Blood. 2009;114(1):174-180.

13. Chan KL, Hickson ID. On the origins of ultra-fine anaphase bridges. Cell Cycle. 2009;8(19):3065-3066.

14. Howlett NG, Taniguchi T, Durkin SG, D’Andrea $\mathrm{AD}$, Glover TW. The Fanconi anemia pathway is required for the DNA replication stress response and for the regulation of common fragile site stability. Hum Mol Genet. 2005;14(5):693-701.

15. Tiu R, Gondek L, O'Keefe C, Maciejewski JP. Clonality of the stem cell compartment during evolution of myelodysplastic syndromes and other bone marrow failure syndromes. Lenkemia. 2007; 21(8):1648-1657.

16. Kastan MB, Bartek J. Cell-cycle checkpoints and cancer. Nature. 2004;432(7015):316-323.

17. Boehrer S, et al. Suppression of the DNA damage response in acute myeloid leukemia versus myelodysplastic syndrome. Oncogene. 2009;28(22):2205-2218.

18. Lo Ten Foe JR, et al. Somatic mosaicism in Fanconi anemia: molecular basis and clinical significance. Eur J Hum Genet. 1997;5(3):137-148. 


\title{
Charting the course across the blood-brain barrier
}

\author{
David Nathanson and Paul S. Mischel
}

\author{
Department of Pathology and Laboratory Medicine and Department of Molecular and Medical Pharmacology, \\ David Geffen School of Medicine, UCLA, Los Angeles, California, USA.
}

\begin{abstract}
The blood-brain barrier (BBB) presents a significant obstacle to delivery of targeted therapies to brain tumors. In this issue of the JCI, Staquicini and colleagues apply an in vivo phage-displayed library of random peptides to identify differentially expressed peptides that can be used to transport targeted agents across the intact $\mathrm{BBB}$. The authors uncover a non-canonical, peptide-mediated iron-mimicry mechanism to induce transport of the transferrin/transferrin receptor complex across the BBB. They then demonstrate the ability of phage-targeting approaches to deliver therapeutic cargo and molecular imaging reporters across the $\mathrm{BBB}$ in an intracranial glioblastoma mouse model.
\end{abstract}

Malignant gliomas are the most common primary brain tumor in adults and one of the most lethal of all cancers. With a median survival rate of one year, they present an almost unparalleled clinical challenge. Whether they arise from the constituent cells of the brain or metastasize from other sites, gliomas are often located in the most functionally important areas of the brain, making complete surgical resection a virtual impossibility. Furthermore, most brain tumors are relatively radio- and chemoresistant, while the surrounding normal brain tissue is relatively sensitive, making attempts at successful treatment resemble Ulysses' frightening effort to pass between Scylla and Charybdis, as described in Homer's Odyssey.

Comprehensive genomic surveys have identified a number of potentially targetable mutations in glioblastoma (GBM), the most common and malignant type of glioma. These mutations, which cluster along the EGFR/PTEN/PI3K, p53, and pRb1 signaling pathways $(1,2)$, have been demonstrated to play a causative role in malignant glioma formation and progression in mouse genetic models $(3,4)$, which suggests that small-molecule inhibitors and antibodies targeting these pathways may play an important role in reshaping the future treatment of patients with GBM. However, delivering these treatments to the brain remains a significant challenge.

Conflict of interest: The authors have declared that no conflict of interest exists.

Citation for this article: J Clin Invest. 2011; 121(1):31-33. doi:10.1172/JCI45758.
The BBB: an obstacle to the delivery of brain tumor treatments

The brain is a privileged site, sheltered from the systemic circulation by the blood-brain barrier (BBB) - a structure composed of endothelial cells, associated astrocytic end-feet processes, perivascular neurons, and pericytes (Figure 1). The endothelial cells are connected by tight junctions that form an almost impenetrable barrier to all compounds except highly lipidized small molecules of less than $400 \mathrm{Da}$. Thus, delivery of the vast majority of therapeutic small molecules to the brain parenchyma is greatly limited. Brain tumors such as GBM may partially disrupt the BBB by inducing large gaps between endothelial cells (5). However, the extent of BBB disruption among individual patients, and/or among various regions within a single tumor, appears to be highly variable. Therefore, the BBB presents a significant obstacle to delivery of targeted brain tumor treatments. Attempts to artificially disrupt the BBB by intra-arterial infusion of hyperosmotic solutions presents one potential therapeutic option, as recently demonstrated by intra-arterial infusion of the VEGFspecific antibody bevacizumab after BBB disruption by mannitol in patients with recurrent malignant glioma (6). However, disruption of the BBB could potentially lead to other serious complications, such as brain edema. Therefore, development of strategies to deliver targeted agents across the $\mathrm{BBB}$ is a critical priority.

Several strategies to get therapeutic molecules across the BBB have recently been studied in malignant glioma patients. These include: (a) transnasal delivery (7); (b) convection-enhanced delivery (CED), whereby a therapeutic agent is continuously infused into the tumor bed under positive pressure to create a pressure gradient, enabling delivery to a larger region of the brain than can be achieved by diffusion (8); (c) packaging drugs and/or interfering RNAs into polyethylene glycolencapsulated liposomes that more readily cross the BBB and show tumor reduction and increase in survival in mice compared with systemic delivery, an approach that can be combined with CED $(9,10)$; (d) use of replication-competent retroviruses to deliver oncolytic therapies (11); and (e) use of mesenchymal (12) or neural stem cells to deliver small molecules, antibodies, or toxic payloads $(13,14)$. Alternatively, other investigators have sought to take advantage of endogenous BBB transporters to increase delivery of targeted agents. There are three main classes of BBB transporters: (a) carrier-mediated transporters, including the glucose and amino acid transporters; (b) active efflux transporters, including $\mathrm{P}$-glycoprotein and the other ABC gene family members; and (c) receptor-mediated transporters, of which transferrin receptor (TfR), insulin receptor, and low-density lipoprotein receptor are the best characterized (15). Attempts to target GBM by delivering EGFR-specific shRNA in PEGylated liposomes bearing insulin receptor- and TfR-specific antibodies in an in vivo model has shown some promise (16). However, these antibody-based approaches have yet to translate into the clinic. Developing the right targeting antibodies to facilitate crossing of the BBB in humans, and uncovering the molecular mechanism by which the process works, remain significant impediments to clinical application.

\section{Phage display to identify differentially expressed peptides that can be used to transport targeted agents across the intact $B B B$}

In this issue of the JCI, Staquicini et al. provide an ingenious alternative strategy to overcoming the obstacle of delivering targeted agents across the BBB, based on 